\title{
Hypoxia: Unique myocardial morphology?
}

\author{
Antonio F. Corno, MD, FRCS, FETCS ${ }^{a}$ \\ Giuseppina Milano, $\mathrm{PhD}^{\mathrm{a}}$ \\ Sandrine Morel, $\mathrm{PhD}^{\mathrm{a}}$ \\ Piergiorgio Tozzi, MD ${ }^{\mathrm{a}}$ \\ Claude Y. Genton, MD ${ }^{\mathrm{a}}$ \\ Michele Samaja, $\mathrm{PhD}, \mathrm{MD}^{\mathrm{b}}$ \\ Ludwig K. von Segesser, MD, FACS, FETCS ${ }^{a}$
}

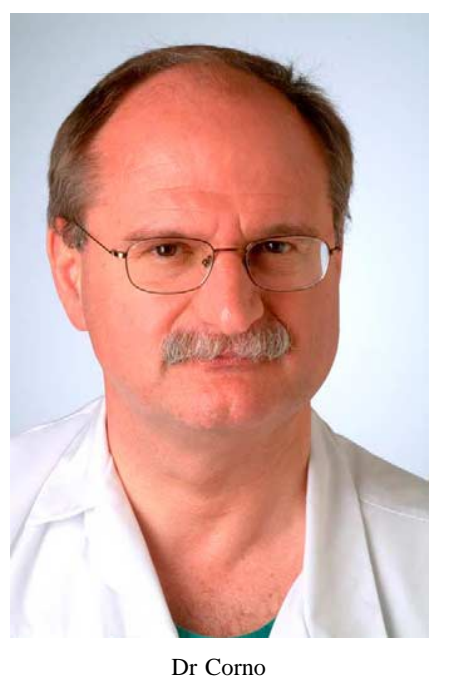

From the Centre Hospitalier Universitaire Vaudois, ${ }^{a}$ Lausanne, Switzerland, and the University of Milan, ${ }^{\mathrm{b}}$ Milan, Italy.

Received for publication March 12, 2003; revisions requested May 22, 2003; accepted for publication June 18, 2003.

Address for reprints: Antonio F. Corno, MD, FRCS, FETCS, Department of Cardiovascular Surgery, Centre Hospitalier Universitaire Vaudois (CHUV), 46 rue du Bugnon, CH 1011, Lausanne, Switzerland (E-mail: Antonio.Corno@chuv.hospvd.ch).

J Thorac Cardiovasc Surg 2004;127:1301-8 $0022-5223 / \$ 30.00$

Copyright () 2004 by The American Association for Thoracic Surgery

doi:10.1016/j.jtcvs.2003.06.012
Objective: The objective of this study was to investigate the effects of chronic and intermittent hypoxia on myocardial morphology.

Methods: Rats randomly divided into 3 groups ( $\mathrm{n}=14$ per group) were exposed to room air $\left(\mathrm{FIO}_{2}=0.21\right)$, chronic hypoxia $\left(\mathrm{FIO}_{2}=0.10\right)$, and intermittent hypoxia (chronic hypoxia with 1 hour per day of room air) for 2 weeks. Weight, blood gas analysis, hematocrit, hemoglobin, red cells, and right and left ventricular pressures were measured. Hearts excised for morphologic examination were randomly divided into 2 groups ( 9 per group for gross morphologic measurements and 5 per group for histologic and morphometric analysis). The weight ratio of right to left ventricles plus interventricular septum, myocyte diameter, cross-sectional area, and free wall thickness in right and left ventricles were measured.

Results: Despite the same polycythemia, the right ventricle pressure $(P<.05)$ and ratio of right to left ventricle pressures $(P<.02)$ were higher after chronic hypoxia than intermittent hypoxia. The ratio of heart weight to total body weight and the ratio of right to left ventricles plus interventricular septum was higher $(P<.01)$ in chronic and intermittent hypoxia than in normoxia. Myocyte diameter was not different between the right and left ventricles in normoxia, whereas right ventricle myocytes were larger than left ventricle myocytes in chronic hypoxia $(P<.05)$ and intermittent hypoxia $(P<.0005)$. There was marked dilatation of right ventricle size $(P<.001)$ and marked reduction of left ventricle $(P<.001)$ size in chronic and intermittent hypoxia compared with normoxia. The total ventricular area (right ventricle plus left ventricle area) remained the same in all groups. The wall thickness ratio in chronic hypoxia and intermittent hypoxia was increased $(P<.001)$ compared with normoxia in the right ventricle but not in the left ventricle.

Conclusions: Intermittent reoxygenation episodes do not induce a lesser ventricular hypertrophic response than observed with chronic hypoxia. The functional myocardial preconditioning consequence of intermittent reoxygenation is not supported by structural differences evident with the available techniques.

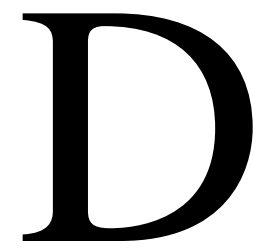

espite conflicting evidence regarding the impact of chronic cyanosis on cardiac surgery outcome, most investigators agree that it does have a deleterious effect overall. ${ }^{1-5}$

In previous experimental studies, we observed the negative effects of exposure to acute ${ }^{6-9}$ and chronic hypoxia ${ }^{10,11}$ on myocardial metabolism and function. In these studies, with the use of a specially designed system of hypoxic chambers, ${ }^{10,11}$ the animals were maintained with continuous exposure to a low oxygen level for 2 weeks, avoiding even short periods of unwanted exposure to room air during feeding, maintenance, and heart isolation. Our study demonstrated that the exposure of the animals to room air for 
even 1 hour per day (intermittent hypoxia) substantially reduced the myocardial effects of low oxygen exposure for the remaining 23 hours per day. ${ }^{11}$ As a consequence, we speculated that all of the previously reported experimental studies using conventional hypoxic chambers (requiring opening the cage for feeding, maintenance, and heart isolation) were in reality dealing with intermittent hypoxia instead of chronic hypoxia.

Because our previous observations have been limited to the effects of hypoxia on myocardial metabolism and function, and relatively little is known about the effects on myocardial structure, we decided to apply our experimental model of chronic and intermittent hypoxia to study the effects on myocardial morphology.

\section{Materials and Methods Animals}

Sprague-Dawley male rats (5 weeks old and weighing 230-250 g at entry in the protocol) were randomly divided into 3 groups $(\mathrm{n}=$ 14 per group): (1) control normoxia exposed to room air $\left(\mathrm{FIO}_{2}=\right.$ $0.21)$, (2) chronic hypoxia $\left(\mathrm{FIO}_{2}=0.10\right)$, and (3) intermittent hypoxia (as for chronic hypoxia, $\mathrm{FIO}_{2}=0.10$, but with exposure to room air for 1 hour per day). The animals in the chronic hypoxia and intermittent hypoxia groups were housed for 2 weeks in our system of normobaric hypoxic chambers previously described. ${ }^{10,11}$ The oxygen tension inside the hypoxic chambers was continuously monitored by an $\mathrm{O}_{2}$ electrode (Servomex Oxygen Analyzer $570 \mathrm{~A}$; Zurich, Switzerland). All animals had free access to water and standard rat chow containing $90 \mathrm{mg} / \mathrm{kg} \alpha$-tocopherol until 24 hours before the experiment. Water and food consumption were assessed every 2 days.

The investigation conforms to the "Guide for the Care and Use of Laboratory Animals," published by the US National Institutes of Health (NIH Publication No. 85-23, revised 1996).

\section{Blood Samples, Ventricular Pressure Measurements, and Heart Isolation}

Rats were weighed and then anesthetized with an intraperitoneal injection of sodium thiopental ( $10 \mathrm{mg} / 100 \mathrm{~g}$ body weight) and 500 units of heparin. Immediately after induction of general anesthesia, a blood sample was collected from the femoral artery for blood gas analysis, hematocrit, hemoglobin, red cell count, and mean red cell volume. After the chest was opened through median sternotomy and longitudinal pericardiotomy, the systolic right ventricular (RV) and left ventricular (LV) pressures were directly measured with a $25 \mathrm{G}$ needle (Terumo; Leuven, Belgium) connected to a pressure transducer (MPC-500; Millar Instruments Inc., Houston, Tex) and recorded.

At this point, the 14 hearts from each group were excised for morphologic examination and randomly distributed into 2 subgroups ( 9 hearts per group for gross morphologic measurements and 5 hearts per group for histologic and morphometric analysis).

\section{Morphologic Measurements}

For morphologic measurements, the hearts were excised, excess water was absorbed on tissue paper, and the heart mass was weighed. The atria were excised, and the free walls of the RV and LV and the septum were dissected free, dried for 48 hours at $90^{\circ} \mathrm{C}$, and weighed separately. RV hypertrophy was assessed from the weight ratio of the RV to LV plus interventricular septum (IS) $(\mathrm{RV} /[\mathrm{LV}+\mathrm{IS}])$.

\section{Histologic and Morphometric Analysis}

The hearts were fixed immediately after excision in $4 \%$ buffered formaldehyde. Two adjacent slices were taken perpendicular to the long axis of the heart immediately under the atrioventricular junction, dehydrated, and embedded in paraffin. Sections $4 \mu \mathrm{m}$ thick were cut on a microtome and stained with hematoxylin-eosin and periodic acid-Schiff. This last stain emphasizes the basal membrane, thus facilitating the microscopic measurement of the myocytes. To determine the presence and degree of hypertrophy, the diameters of 60 individual myocytes in each ventricle of all hearts were measured (Nikon Eclipse E 800; Zürich, Switzerland) by using a digital camera and Image-Pro Plus (MediaCybernetics; Carlsbad, Calif) (Figure 1). To avoid orientation artifacts, the smallest diameter measured in individual myocytes with a visible nucleus determined the retained value.

The cross-sectional area of both ventricles and the thickness of their free walls were also measured.

\section{Statistics}

Data are expressed as mean \pm SE. Significant differences among the 3 groups were detected by performing the 1-way analysis of variance test. Where significant, the differences between selected pairs of data were tested using the Fischer comparison procedure (StatView; Abacus Concepts, Berkeley, Calif).

\section{Results}

\section{Blood Values and Ventricular Pressures}

The values of hematocrit, hemoglobin, red blood cells, mean red cell volume, arterial $\mathrm{Po}_{2}, \mathrm{PCO}_{2}, \mathrm{O}_{2}$ saturation, and $\mathrm{pH}$, amount of ingested food and water per day, and final body and heart weights obtained at the end of the 2 weeks of observation are reported in Table 1. The ratios of systolic $\mathrm{RV}$ to $\mathrm{LV}$ pressures and $\mathrm{RV}$ to $\mathrm{LV}$ systolic pressures are reported in Table 2 . The data related to the daily water and food intake were very similar to our previously reported observations, as were the final body and heart weights. ${ }^{10,11}$ The final body weight observed in the control normoxic group $(351 \pm 13 \mathrm{~g})$ confirms that the rats were still in the neonatal growing age. Despite the same degree of polycythemia observed in both hypoxic groups, there was a significant difference between chronic and intermittent hypoxia with systolic RV pressure and the ratio of RV to LV pressures both higher after chronic hypoxia compared with intermittent hypoxia $(P<.05$ and $P<.02$, respectively).

\section{Heart Weight and RV Hypertrophy}

The graphics in Figure 2 represent the effects of exposure to hypoxia on heart weight and the ratio of the heart weight to 

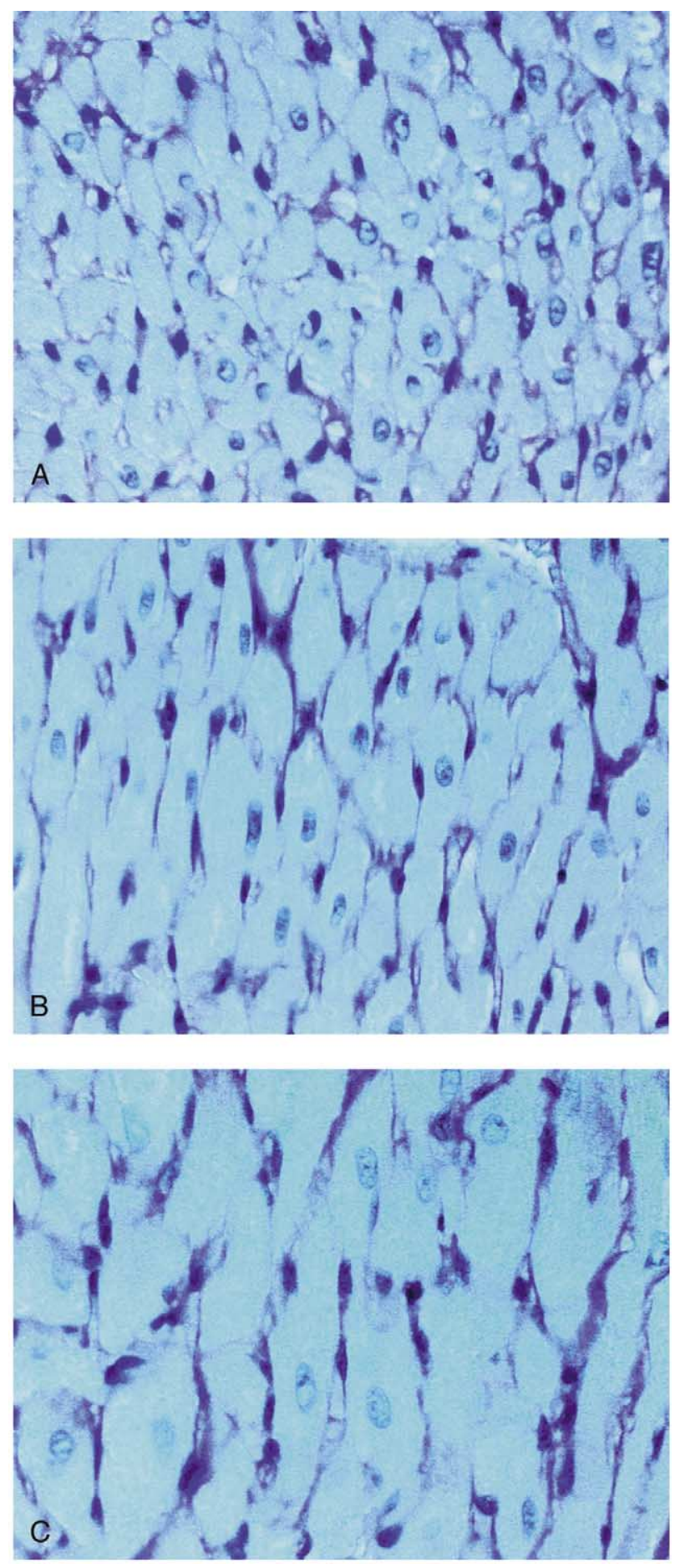

Figure 1. RV myocytes after normoxia (A), chronic hypoxia (B), and intermittent hypoxia (C) (images taken under light microscopy $[\times 400]$ using a digital camera).

the body weight (an index of cardiac hypertrophy) at the end of 2 weeks. Although the heart weight was less in the chronic hypoxic group than in the normoxic or intermittent
TABLE 1. Blood values, intakes, and weights of animals

\begin{tabular}{|c|c|c|c|}
\hline Parameters & Normoxia & $\begin{array}{l}\text { Chronic } \\
\text { hypoxia }\end{array}$ & $\begin{array}{c}\text { Intermittent } \\
\text { hypoxia }\end{array}$ \\
\hline Arterial $\mathrm{Po}_{2}, \mathrm{~mm} \mathrm{Hg}$ & $58.0 \pm 2.8$ & $35.3 \pm 1.1^{*}$ & $34.5 \pm 2.3^{*}$ \\
\hline Arterial $\mathrm{PCO}_{2}, \mathrm{~mm} \mathrm{Hg}$ & $49.0 \pm 4.9$ & $28.9 \pm 1.4^{*}$ & $26.9 \pm 1.1^{*}$ \\
\hline $\begin{array}{l}\text { Arterial } \mathrm{O}_{2} \text { saturation, } \\
\%\end{array}$ & $81 \pm 5$ & $53 \pm 7^{*}$ & $54 \pm 7^{*}$ \\
\hline Arterial pH & $7.39 \pm 0.02$ & $7.41 \pm 0.01$ & $7.41 \pm 0.01$ \\
\hline Hematocrit, \% & $45 \pm 1$ & $68 \pm 1^{*}$ & $68 \pm 1^{*}$ \\
\hline Hemoglobin, g/L & $139.3 \pm 2.1$ & $209.4 \pm 3.1^{*}$ & $209.3 \pm 1.8^{*}$ \\
\hline $\begin{array}{l}\text { Red blood cells/ } \mu \mathrm{L} / \\
\quad 1000\end{array}$ & $7.1 \pm 0.1$ & $9.9 \pm 0.2^{*}$ & $9.7 \pm 0.1^{*}$ \\
\hline $\begin{array}{l}\text { Mean red cell volume, } \\
\text { f1 }\end{array}$ & $63 \pm 1$ & $70 \pm 1^{*}$ & $70 \pm 1^{*}$ \\
\hline Ingested food, g/d & $30.7 \pm 1.3$ & $21.3 \pm 1.9^{*}$ & $17.9 \pm 1.1^{*}$ \\
\hline Ingested water, $\mathrm{mL} / \mathrm{d}$ & $50.4 \pm 4.9$ & $19.9 \pm 0.9^{*}$ & $22.5 \pm 0.7^{*} \dagger$ \\
\hline Final body weight, g & $351 \pm 13$ & $187 \pm 4^{*}$ & $250 \pm 7^{*} \dagger$ \\
\hline Heart weight, mg & $1312 \pm 43$ & $1111 \pm 50^{*}$ & $1407 \pm 56 \dagger$ \\
\hline
\end{tabular}

*Significant difference versus normoxia, $P<.0001$.

tSignificant difference versus chronic hypoxia, $P<.05$.

TABLE 2. Ventricular pressures

\begin{tabular}{lccc}
\hline Parameters & Normoxia & $\begin{array}{c}\text { Chronic } \\
\text { hypoxia }\end{array}$ & $\begin{array}{c}\text { Intermittent } \\
\text { hypoxia }\end{array}$ \\
\hline $\begin{array}{c}\text { RV systolic pressure, } \\
\text { mm Hg }\end{array}$ & $28 \pm 1$ & $53 \pm 6^{*}$ & $31 \pm 1^{*}$ \\
$\begin{array}{c}\text { LV systolic pressure, } \\
\text { mm Hg }\end{array}$ & $42 \pm 2$ & $41 \pm 4$ & $46 \pm 6$ \\
$\begin{array}{l}\text { RV/LV systolic } \\
\text { pressures ratio }\end{array}$ & $0.67 \pm 0.01$ & $1.28 \pm 0.09 \dagger$ & $0.69 \pm 0.10 \ddagger$ \\
\hline
\end{tabular}

$R V$, Right ventricle; $L V$, left ventricle.

*Significant difference versus normoxia, $P<.05$.

†Significant difference versus normoxia, $P<.005$.

$¥$ Significant difference versus chronic hypoxia, $P<.05$.

hypoxic groups, the heart weight to total body weight ratio was significantly higher in the chronic and intermittent hypoxic groups than in the normoxic group. This observation, correlated with the substantially reduced body weight observed in rats after 2 weeks of chronic hypoxia, confirms the higher degree of ventricular hypertrophy observed in this group of animals.

The ratio of $\mathrm{RV} /(\mathrm{LV}+\mathrm{IS})$, a reliable index of $\mathrm{RV}$ hypertrophy, was significantly increased in both the chronic and intermittent hypoxic groups (Figure 3).

\section{Morphometric Evaluations}

The evaluation of the morphometric changes in the RV and $\mathrm{LV}$ was performed using horizontal slices of hearts from all 3 groups (Figure 4).

Myocyte diameter. In Figure 5, the effects of chronic and intermittent hypoxia on myocyte diameter in the RV and LV are represented. Compared with normoxic hearts, exposure to both chronic and intermittent hypoxia caused a 
heart weight

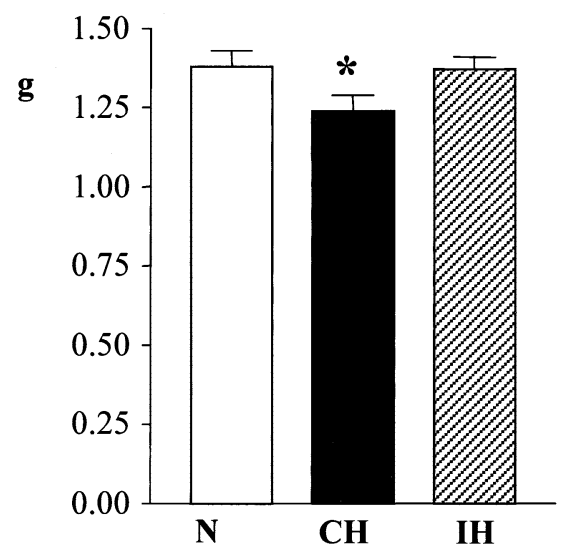

heart weight / body weight

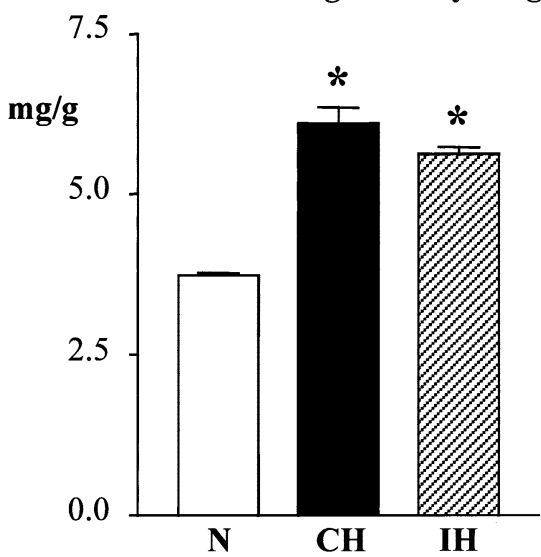

Figure 2. Heart weight and ratio of heart weight to total body weight of hearts exposed to $\mathbf{N}$, $\mathbf{C H}$, and IH. *Significant difference versus normoxia, $P$ less than .01, 1-way analysis of variance (ANOVA). N, Normoxia; $\mathrm{CH}$, chronic hypoxia; $I H$, intermittent hypoxia.

$\mathbf{R V} /(\mathbf{L V}+\mathbf{I S})$

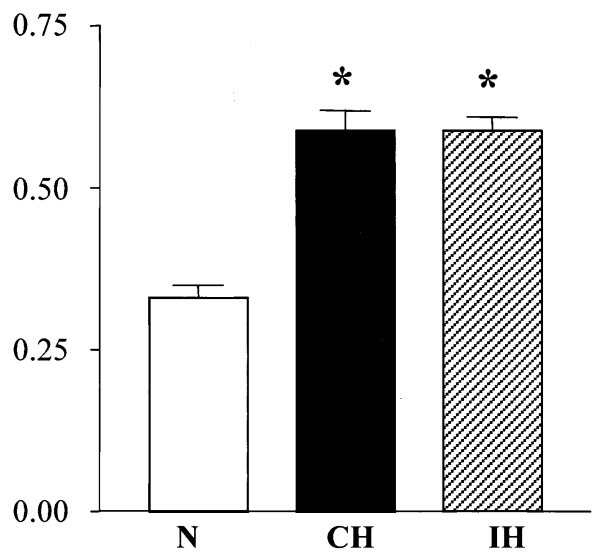

Figure 3. The ratio $\mathrm{RV} /(\mathrm{LV}+\mathrm{IS})$ of hearts exposed to $\mathrm{N}, \mathrm{CH}$, and IH. *Significant difference versus normoxia, $P$ less than .001, 1-way ANOVA. $N$, Normoxia; $\mathrm{CH}$, chronic hypoxia; $\boldsymbol{I H}$, intermittent hypoxia; $R V /(L V+I S)$, right ventricle to left ventricle plus interventricular septum.

significant and similar increase in myocyte diameters in the $\mathrm{RV}$, but not in the LV. There were no statistically significant differences in myocyte diameter between the RV and LV in normoxic hearts, whereas the RV myocytes were larger than the LV myocytes in both chronic hypoxic $(P<.05)$ and intermittent hypoxic $(P<.0005)$ hearts.

Cross-sectional ventricular area. Figure 6 shows the effects of exposure to chronic and intermittent hypoxia on a cross-sectional area of the RV and LV at the end of 2 weeks. There is a marked dilatation of RV size and a marked reduction of LV size in both chronic and intermittent hypoxic hearts compared with normoxic hearts.

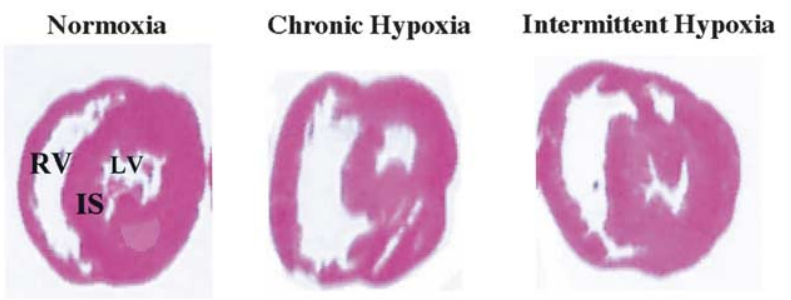

Figure 4. Myocardial transversal slices. IS, Interventricular septum; $L V$, left ventricle; $R V$, right ventricle.

Notably, the total ventricular area (RV area $+\mathrm{LV}$ area) remained the same in all groups $(16.27 \pm 2.36,16.47 \pm$ 2.08 , and $16.40 \pm 1.88 \mathrm{~mm}^{2}$ in normoxic, chronic hypoxic, and intermittent hypoxic hearts, respectively.)

Ventricular wall thickness ratio. In Figure 7, the consequences of exposure to chronic or intermittent hypoxia are reported with regard to the wall thickness ratio for the $\mathrm{RV}$ and LV. The RV wall thickness ratio in chronic and intermittent hypoxic hearts was significantly increased compared with normoxic hearts. In contrast, exposure to chronic and intermittent hypoxia did not induce any difference for LV wall thickness ratio compared with normoxia.

\section{Discussion}

In experimental studies on the effects of hypoxia, several variables need to be taken in consideration, including the animal species, age at entry into the protocol, model used to produce hypoxemia, intensity and duration of hypoxemia before the experimental study, and precise nature of the experimental design evaluating the myocardial response. $^{12,13}$ 

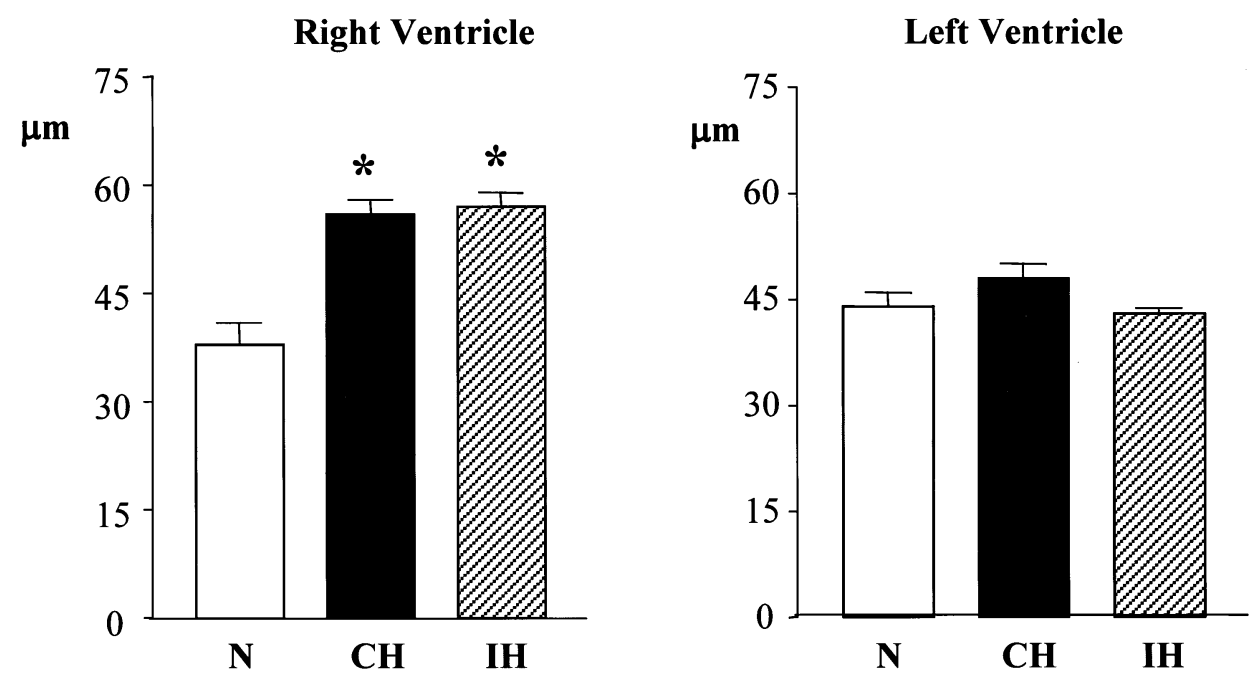

Figure 5. Myocyte diameter in hearts exposed to $\mathbf{N}, \mathrm{CH}$, and IH. *Significant difference versus normoxia, $\boldsymbol{P}$ less than .001, 1-way ANOVA. N, Normoxia; $C H$, chronic hypoxia; IH, intermittent hypoxia.

\section{Right Ventricle}

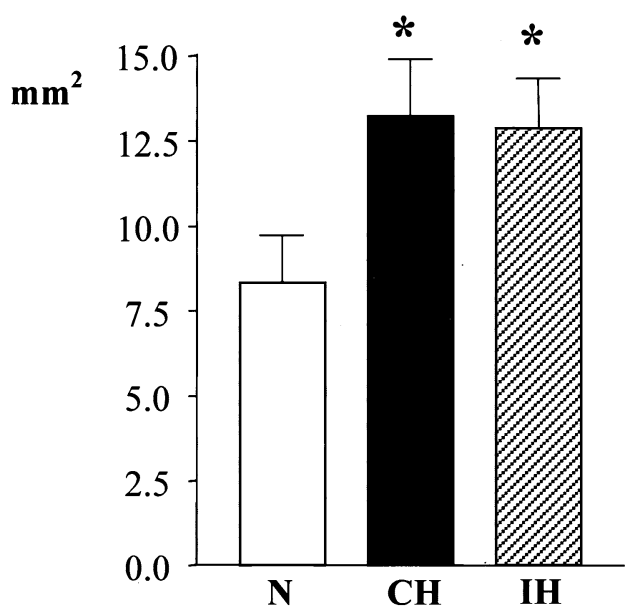

Left Ventricle

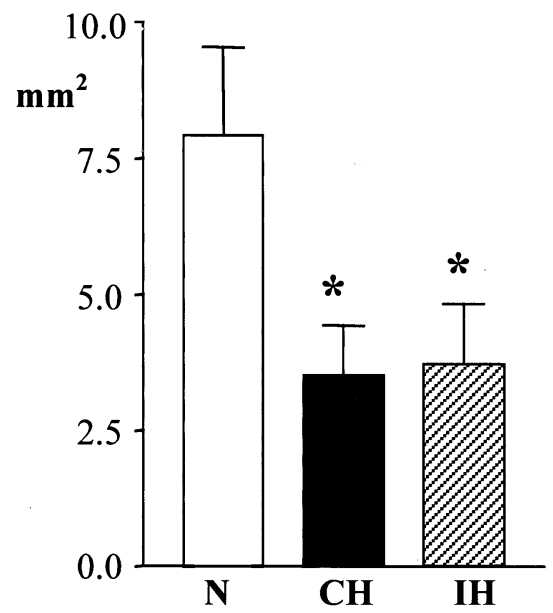

Figure 6. Cross-sectional area in hearts of rats exposed to $\mathrm{N}, \mathrm{CH}$, and $\mathrm{IH}$. *Significant difference versus normoxia, $P$ less than .001, 1-way ANOVA. $N$, Normoxia; $C H$, chronic hypoxia; $I H$, intermittent hypoxia.

Despite 2 recent reports of validated mathematical mod$\mathrm{els},{ }^{14,15}$ the use of protocols with animal models still remains a reliable system to evaluate the consequences of oxygen shortage.

The $\mathrm{FIO}_{2}$ of 0.10 selected for this study of chronic and intermittent hypoxia is equivalent to the exposure to 5.500 m altitude. ${ }^{16}$

The potential limits of our model in terms of animal species, age, and type of environmental hypoxia have already been recognized. ${ }^{10}$ Nevertheless, with our system of hypoxic cages we were able to distinguish between the real "continuous chronic hypoxia" and what erroneously has always been called "chronic hypoxia" by other researchers. We previously demonstrated that the situation realized a form of intermittent hypoxia with negative effects of much lesser degree on the myocardial metabolism and function than the continuous chronic hypoxia. ${ }^{11}$

Because of these observations, we evaluated the effects of chronic and intermittent hypoxia on the myocardial structure to find out whether there were structural reasons to justify the metabolic and functional differences previously observed between chronic and intermittent hypoxia. 
RV/(LV+IS)

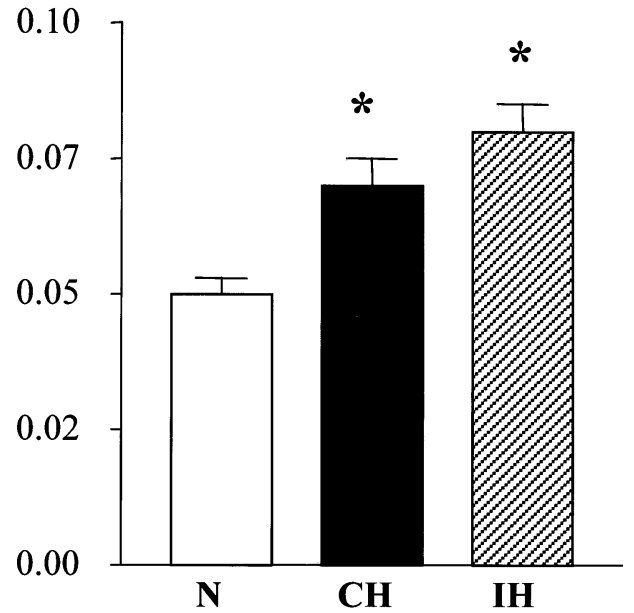

LV/(RV+IS)

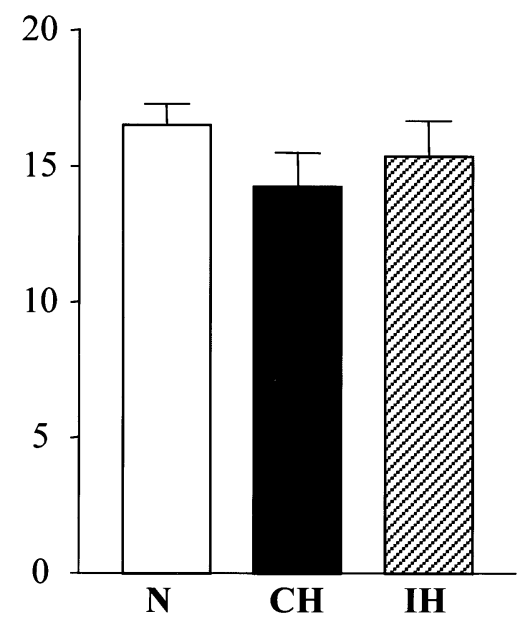

Figure 7. RV and LV wall thickness ratios in hearts exposed to $\mathrm{N}, \mathrm{CH}$, and IH. *Significant difference versus normoxia, $P$ less than .001, 1-way ANOVA. $N$, Normoxia; $C H$, chronic hypoxia; $I H$, intermittent hypoxia.

\section{Myocardial Hypertrophy}

With regard to the myocardial structure and the response to the stimuli, cardiac growth can be mediated by an increase in the number of myocytes (hyperplasia) or by enlargement of preexisting myocytes (hypertrophy). In the embryonic and fetal stages, cardiac growth is primarily because of hyperplasia, and this process persists during the neonatal life. The mitotic activity and the potential for hyperplasia of myocytes are probably lost between 3 and 6 months of age, and the cardiac growth remains only because of hypertrophy. In the neonatal heart, a pressure overload induces myocyte hyperplasia, hypertrophy, and capillary proliferation. In adult hearts, a pressure overload induces only myocyte hypertrophy without capillary proliferation, ${ }^{17}$ whereas intermittent exposure to hypoxia with a repeated long interval of reoxygenation fails to determine changes at myocyte and capillary density. ${ }^{18}$ Hypertrophy has been assumed to be the only form of myocyte growth in the adult heart, because the cardiac myocytes are believed to be terminally differentiated cells and have been compared with neurons because of their inability to regenerate and replace damaged myocardium. Ventricular pressure overload induces concentric hypertrophy with an increase in wall thickness without chamber enlargement. ${ }^{19}$ Wall thickening is the result of an increase in myocyte diameter without changes in the number of myocytes in the wall or in the aggregate number of cells in the entire ventricle. ${ }^{19}$ The combination of these events leads to an increase in the ventricular mass to chamber volume ratio. ${ }^{20}$

\section{Ventricular Interdependence}

Despite the fact that the heart has always been considered constituted by 2 muscular pumps (right and left) connected in series to one another by the pulmonary and systemic circulations, with the ventricular septum as the key structure of interacting ventricular function, the unique structural and functional entity of the myocardium with spiral organization was recently revealed by Buckberg. ${ }^{21}$ The functional interaction of RV and LV therefore occurs not only because of their arrangements in series but also because of their structural spiral features. ${ }^{21}$ Changes in size and function of either ventricle may influence the performance of the other ventricle, and this is particularly evident in the neonatal myocardium. $^{22}$

\section{Hypoxia and Myocardial Hypertrophy: Hemodynamic Factors}

Vasoconstriction and structural remodeling induced by chronic hypoxia in the pulmonary circulation ${ }^{23-27}$ alter the pulmonary hemodynamics and lead to pulmonary hypertension, with a selective hypertrophy of the RV. In addition to prolonged pulmonary vasoconstriction and systemic vasodilatation, exposure to chronic hypoxia stimulates the erythropoiesis, resulting in polycythemia and an associated increase in blood viscosity, which may also contribute to pulmonary hypertension. ${ }^{28,29}$

Our experimental results showed that exposure to both chronic and intermittent hypoxia induces a similar degree of RV hypertrophy, as evidenced by the greater weight ratio of 
the $\mathrm{RV} /(\mathrm{LV}+\mathrm{IS})$, increased free wall thickness, and increased diameter of RV myocytes.

The RV hypertrophy is a consequence of pulmonary hypertension. Our results indicate that the degree of RV hypertrophic response induced by chronic hypoxia is not modified by repeated reoxygenation episodes (intermittent hypoxia).

The exposure of the entire myocardium to the same oxygen shortage with chronic and intermittent hypoxia, associated with the same degree of polycythemia observed in both hypoxic groups, and therefore with probably the same degree of increased viscosity on the systemic and pulmonary circulations, does not induce LV hypertrophy. In fact, the measured diameter of LV myocytes in both chronic and intermittent hypoxic hearts was not different from that in normoxic hearts.

Therefore, the structural differences observed between the RV and LV in terms of ventricular hypertrophy have to be a consequence of the different hemodynamic situation determined in both ventricles by the chronic oxygen shortage. ${ }^{27}$ This speculation has been only partially confirmed in our study by the values of systolic pressures measured in the ventricular chambers at the end of the observation period, with the RV pressure significantly increased to supra-systemic values only in the chronic hypoxic hearts. In the group of animals exposed to intermittent hypoxia, the RV systolic pressure was higher than in the normoxic control group, but it remained well below the systemic values, as if intermittent episodes of reoxygenation are sufficient to reduce the pulmonary vasoconstriction induced by hypoxia.

Under physiologic conditions, systolic and diastolic LV loading exceeds that of the RV, and the ventricular septum becomes concave with respect to the center of the LV cavity, resulting in a relatively circular short-axis crosssectional LV profile. ${ }^{30}$ In contrast, exposure to chronic hypoxia induces a RV pressure overload and a LV filling impairment despite a normal systolic phase because of a septal leftward shift, without significant impairment of the LV systolic performance per se. Despite relative underfilling of the LV in RV pressure overload, the resting LV ejection fraction is preserved. ${ }^{31}$

In our experimental study, the exposure to either chronic or intermittent hypoxia induced a significant increase of the $\mathrm{RV}$ cross-sectional area with a correspondent reduction of LV cross-sectional area in comparison with control normoxic hearts. We can explain this difference between the $\mathrm{RV}$ and LV cross-sectional area with the shift of the IS toward the LV cavity because of the RV hypertension, a consequence of pulmonary vasoconstriction and polycythemia both increasing the pulmonary vascular resistance. Because polycythemia also affected the systemic vascular resistance, we have to confirm the previous observation that hypoxia determines pulmonary vasoconstriction with simultaneous systemic vasodilatation.

What we are not able to explain are the similar structural findings observed in chronic and intermittent hypoxia with regard to the cross-sectional ventricular area and degree of RV hypertrophy (free wall thickness, ventricular weight, and myocyte diameter) in correspondence with a significantly different hemodynamic pattern of systolic pressure in the 2 ventricles. We can only speculate that the period of 2 weeks is not enough to determine definitive structural differences; in 2 weeks the myocytes synthesized what is needed for the process of hypertrophy, and this process is already started (as proved by the increased diameter of the myocytes) but is not yet finished.

Even more interesting is the observation that the total ventricular area remained the same in all groups, irrespective of the changes of separate ventricles. If this somehow confirms the unistructural spiral vision of Buckberg ${ }^{21}$ and the hypothesis of the shift of the IS, we have to admit that the LV filling was impaired by the pressure elevation in the $\mathrm{RV}$. This observation is in agreement with the impaired LV function previously observed and reported. ${ }^{10,11}$ As a matter of fact, the rats exposed to hypoxia had reduced physical activity in comparison with the normoxic control group, as documented by the significantly reduced amount of water and food intake. ${ }^{10,11}$

\section{Conclusion}

With the results of our study, we can speculate that the exposure to intermittent reoxygenation episodes does not influence the ventricular hypertrophic response observed with exposure to chronic hypoxia. The metabolic and functional differences previously observed, ${ }^{11}$ with better LV performance after exposure to intermittent hypoxia, may be the result of the reduced hemodynamic impairment obtained with intermittent reoxygenation or a form of myocardial protection not evident at the structural level with the available techniques. The fact that in the present experimental study we failed to demonstrate structural differences between the intermittent and chronic hypoxic groups represents a stimulus to perform further studies to investigate the differences at the ultrastructural and micromolecular level.

\section{References}

1. Silverman NA, Kohler J, Levitsky S, Pavel DG, Fang RB, Feinberg H. Chronic hypoxemia depresses global ventricular function and predisposes the depletion of high-energy phosphates during cardioplegic arrest: implications for surgical repair of cyanotic congenital heart defects. Ann Thorac Surg. 1984;37:304-8.

2. Najm HK, Wallen WJ, Belanger MP, Williams WG, Coles JG, Van Arsdell GS, et al. Does the degree of cyanosis affect myocardial adenosine triphosphate levels and function in children undergoing surgical procedures for congenital heart disease? J Thorac Cardiovasc Surg. 2000;119:515-24.

3. Imura H, Caputo M, Pawade A, Angelini GD, Suleiman MS. Age- 
dependent and hypoxia-related differences in myocardial protection during pediatric open heart surgery. Circulation. 2001;103:1551-6.

4. Hovels-Gurich HH, Schumaker K, Vazquez-Jimenez JF, Qing M, Huffmeier U, Buding B, et al. Cytokine balance in infants undergoing cardiac operation. Ann Thorac Surg. 2002;73:601-8.

5. Modi P, Imura H, Caputo M, Pawade A, Angelini GD, Suleiman MS. Cardiopulmonary bypass-induced myocardial reoxygenation injury in pediatric patients with cyanosis. J Thorac Cardiovasc Surg. 2002;124: 1035-6.

6. Corno AF, Laks H, Davtyan HG, Flynn WM, Clayton CJ, Drinkwater DC. Acute hypoxia: effects on isolated neonatal heart. Clin Res. 1986;3:5A.

7. Corno AF, Motterlini R, Brenna L, Santoro F, Samaja M. Ischaemia/ reperfusion in the posthypoxaemic re-oxygenated myocardium: haemodynamic study in the isolated perfused rat heart. Perfusion. 1993; 8:113-8.

8. Samaja M, Casalini S, Allibardi S, Corno AF, Chierchia S. Regulation of bioenergetics in $\mathrm{O}_{2}$-limited isolated rat hearts. J Appl Physiol. 1994;77:2530-6.

9. Milano G, Corno AF, De Jong JW, von Segesser LK, Samaja M. Tolerance of isolated rat hearts to low-flow ischemia and hypoxia of increasing duration: protective role of down-regulation and ATP during ischemia. Mol Cell Biochem. 2001;226:141-51.

10. Corno AF, Milano G, Samaja M, Tozzi P, von Segesser LK. Chronic hypoxia: a model for cyanotic heart defects. $J$ Thorac Cardiovasc Surg. 2002;124:105-12.

11. Milano G, Corno AF, Lippa S, von Segesser LK, Samaja M. Chronic and intermittent hypoxia induce different degrees of myocardial tolerance to hypoxia-induced dysfunction. Exp Biol Med. 2002;227:38997.

12. Samaja M, Corno AF. The isolated perfused rat heart as experimental model to assess the genesis of myocardial reoxygenation injury. Cardiovasc Engineering. 1998;3:120-6.

13. Huddleston CB. How do you study blue? J Thorac Cardiovasc Surg. 2002;124:14-5.

14. Ursino M, Magosso E. Acute cardiovascular response to isocapnic hypoxia. I. A mathematical model. II. Model validation. Am J Physiol Heart Circ Physiol. 2000;279:H149-75.

15. Yi CS, Fogelson AL, Keener JP, Peskin CS. A mathematical study of volume shifts and ionic concentration changes during ischemia and hypoxia. J Theor Biol. 2003;220:83-106.

16. Samaja M. Blood gas transport at high altitude. Respiration. 1997;64: $422-8$.
17. Di Donato RM, Fujii AM, Jonas RA, Castaneda AR. Age-dependent ventricular response to pressure overload. J Thorac Cardiovasc Surg. 1992;104:713-22.

18. Rakusan K, Ehrenburg IV, Gulyaeva NV, Tkatchouk EN. The effect of intermittent normobaric hypoxia on myocardial structure in rats. Hypoxia Medical J. 1997;3:3-8.

19. Anversa P, Ricci R, Olivetti G. Quantitative structural analysis of the myocardium during physiologic growth and induced cardiac hypertrophy: a review. J Am Coll Cardiol. 1986;7:1140-9.

20. Grossman W, Carabello BA, Gunther S, Fifer MA. Ventricular wall stress and the development of cardiac hypertrophy and failure. In: Alpert NR, editor. Perspectives in cardiovascular research: myocardial hypertrophy and failure. New York: Raven Press; 1983. p. 1-18.

21. Buckberg GD. Basic science review: the helix and the heart. $J$ Thorac Cardiovasc Surg. 2002;124:863-83.

22. Friedman WF. The intrinsic physiologic properties of the developing heart. Prog Cardiovasc Dis. 1972;15:87-111.

23. Dingemanns KP, Wagenvoort CA. Pulmonary arteries and veins in experimental hypoxia. Am J Pathol. 1978;93:353-68.

24. Morrell NW, Atochina EN, Morris KG, Danilov SM, Stenmark KR. Angiotensin converting enzyme expression is increased in small pulmonary arteries of rats with hypoxia-induced pulmonary hypertension. J Clin Invest. 1995;96:1823-33.

25. Shaul PW, Kinane B, Farrar MA, Buja LM, Magness RR. Prostacyclin production and mediation of adenylate cyclase activity in the pulmonary artery. Alterations after prolonged hypoxia in the rat. $J$ Clin Invest. 1991;88:447-55.

26. Blumberg FC, Lorenz C, Wolf K, Sandner P, Riegger GAJ, Pfeifer M. Increased pulmonary prostacyclin synthesis in rats with chronic hypoxic pulmonary hypertension. Cardiovasc Res. 2002;55:171-7.

27. Rabinovitch M, Gamble W, Nadas AS, Miettinen OS, Reid L. Rat pulmonary circulation after chronic hypoxia: hemodynamic and structural features. Am J Physiol. 1979;236:818-27.

28. Barer GR, Bee D, Wach RA. Contribution of polycythemia to pulmonary hypertension in simulated high altitude rats. J Physiol (Lond). 1983;336:27-8.

29. Winslow RM, Samaja M, West JB. Red cell function at extreme altitudes on Mount Everest. J Appl Physiol. 1984;56:109-16.

30. Lima JAC, Guzman PA, Yin FCP. Septal geometry in the unloaded living human heart. Circulation. 1986;74:463-8.

31. Louie EK, Lin SS, Reynertson SI, Brundage BH, Levitsky S, Rich S. Pressure and volume loading of the right ventricle have opposite effects on left ventricle ejection fraction. Circulation. 1995;92:819-24. 